\title{
Comparison of first-line chemotherapy regimens for ovarian carcinosarcoma: a single institution case series and review of the literature
}

\author{
Melissa Brackmann ${ }^{1}$, Marina Stasenko ${ }^{1}$, Shitanshu Uppal ${ }^{1}$, Jake Erba ${ }^{1}$, R. Kevin Reynolds ${ }^{1}$ and Karen McLean ${ }^{1} 2^{*}$ (D)
}

\begin{abstract}
Background: The optimal first-line chemotherapy for ovarian carcinosarcoma has not yet been determined. We therefore sought to determine the progression-free survival (PFS) and overall survival (OS) for patients with ovarian carcinosarcoma treated at our institution with different first-line chemotherapy regimens.

Methods: This single-institution, retrospective analysis included all patients with ovarian or primary peritoneal carcinosarcoma diagnosed from September 1996 to July 2017. Kaplan Meier analysis with a log-rank Mantel-Cox test was used to compare PFS and OS between treatment groups, and a $p$-value of $<0.05$ was considered statistically significant.

Results: Thirty-one patients met inclusion criteria: two patients were stage IC, 5 were stage II, 21 were stage III, and 3 were stage IV. The median PFS and OS for all stages was 9.3 and 19.7 months respectively. Fifteen patients (48\%) received carboplatin/paclitaxel as first therapy, 7 (23\%) received ifosfamide/paclitaxel, 6 (19\%) received a different regimen, and $3(10 \%)$ did not receive chemotherapy. Patients treated with carboplatin/paclitaxel had a statistically significant longer PFS when compared to those receiving ifosfamide/paclitaxel (17.8 vs. 8.0 months, $p=0.025)$. OS was similar between all comparisons.

Conclusions: In summary, in our cohort of ovarian carcinosarcoma patients, median PFS is longer in patients treated with carboplatin/paclitaxel compared to ifosfamide/paclitaxel. Overall survival was similar for all treatment groups, potentially due to subsequent treatment crossover. Given the rarity and aggressive nature of this tumor, further study into optimal first-line chemotherapy is warranted.
\end{abstract}

Keywords: Ovarian carcinosarcoma, MMMT, Chemotherapy, Outcomes, Carboplatin, Ifosfamide

\section{Background}

Ovarian carcinosarcomas, also known as malignant mixed Mullerian tumors (MMMT), represent 1\% of all histologic subtypes of ovarian cancers and remain poorly understood [1]. While ovarian carcinosarcomas were once thought to be "collision tumors" with the presence of synchronous epithelial carcinoma and stromal sarcoma components, current data suggest they are de-

\footnotetext{
* Correspondence: karenmcl@umich.edu

'Division of Gynecologic Oncology, Department of Obstetrics and Gynecology, University of Michigan, Ann Arbor, USA

${ }^{2}$ University of Michigan, 1500 E. Medical Center Drive, Ann Arbor, MI 48109-5276, USA
}

differentiated carcinomas with cells that have a sarcomatous appearance and also cells with a carcinomatous of diagnosis and a $60 \%$ overall recurrence rate $[1,3]$. A review of the Surveillance, Epidemiology, and End Results (SEER) Program data from 1998 to 2009 demonstrated that patients with ovarian carcinosarcoma have consistently poorer prognosis than those with highgrade papillary serous carcinoma of the ovary. This study consisted of more than 14,000 ovarian cancer patients, approximately $9 \%$ of whom had ovarian carcinosarcoma, 
and the five-year disease specific survival rate was $28.2 \%$ for carcinosarcoma patients compared to $38.4 \%$ for high-grade papillary serous carcinoma patients $(p<$ 0.001) [4]. Several additional single institution studies have also demonstrated that ovarian carcinosarcoma patients have lower response rates to chemotherapy and worse overall and disease-specific survival [4-7]. Given the low incidence of these tumors, prospective trials of chemotherapeutic approaches have been difficult to perform [8]. Thus, the preferred first-line chemotherapy for ovarian carcinosarcoma patients remains unknown.

Data guiding chemotherapy for the treatment of ovarian carcinosarcoma is largely extrapolated from studies on the treatment of uterine carcinosarcoma. Historically, ovarian carcinosarcomas were often treated with the same first-line chemotherapy as uterine carcinosarcomas, ifosfamide/paclitaxel [9, 10]. Additionally, multiple phase II studies investigating the use of carboplatin/paclitaxel in uterine carcinosarcoma demonstrated efficacy with this regimen [11, 12]. These studies have formed the basis of the Gynecologic Oncology Group (GOG) study 261, a phase III randomized, controlled trial comparing ifosfamide/paclitaxel to carboplatin/paclitaxel in the treatment of gynecologic carcinosarcomas, which allowed enrollment of patients with uterine or ovarian primary tumor sites.

Despite the above studies investigating chemotherapy treatment options for uterine carcinosarcoma, there are no published prospective studies looking at treatment options for ovarian carcinosarcoma. Small case series have reported on the efficacy of these and other regimens in the treatment of ovarian carcinosarcoma [5, 13-17]. We sought to determine the progression-free survival (PFS) and overall survival (OS) for patients with ovarian and primary peritoneal carcinosarcoma treated at our institution comparing different first-line chemotherapy regimens. Given the historical use of ifosfamide/paclitaxel and carboplatin/paclitaxel, we sought to compare these two regimens specifically as well as to compare platinum-containing regimens to nonplatinum-containing regimens.

\section{Methods}

This single-institution, retrospective study investigated patients with a pathologic diagnosis of ovarian, tubal, or primary peritoneal carcinosarcoma from September 1996 to July 2017. This study was approved by the University of Michigan Institutional Review Board (IRB \# HUM00099459). Thirty-one patients with a histologic diagnosis of ovarian or primary peritoneal carcinosarcoma were identified (all cases were reviewed by a gynecologic pathologist). Baseline demographic information including age at diagnosis, stage, extent of cytoreduction (with optimal debulking defined as less than two centimeters of residual disease), and race were abstracted from each patient's medical chart. First-line chemotherapy regimen was determined. Date of diagnosis was defined as date of primary debulking surgery or the date of diagnostic biopsy in the case of patients for whom debulking surgery was not undertaken. Progression-free survival (PFS) was defined as time from diagnosis to first evidence any of the following: appearance of new disease via radiographic imaging or clinical exam, elevation in CA125 above the normal range, or patient death from any cause. Overall survival (OS) was defined as months from diagnosis to patient death or last contact.

For statistical analysis, results were analyzed with standard descriptive and inferential statistical methods using SPSS Statistics 22. Mean age at diagnosis was compared using a one-way ANOVA. For categorical demographic variables, a chi-squared test was performed. Grubbs' test for outliers was applied. Kaplan Meier curves were plotted for PFS and OS by initial chemotherapy regimen, and a log-rank Mantel-Cox test was used to compare PFS and OS between groups. Regression modeling was not performed due to small sample size. A $p$-value of $<0.05$ was deemed statistically significant for all comparisons.

\section{Results}

Thirty-one patients met inclusion criteria (Table 1). All patients were Caucasian race, and the mean age at diagnosis was 65.3 years (range $36-89$ years). Twenty-nine patients had primary surgical debulking attempted, with optimal debulking achieved in 19 cases, suboptimal debulking in 9 cases and one case for which residual disease could not be determined from the medical record. Two patients did not undergo primary surgical debulking owing to their advanced stage and poor functional status at the time of diagnosis. With regard to stage at diagnosis, 7 patients were stage IC or II at diagnosis (22\%), 21 patients were stage III (68\%), and 3 patients were stage IV (10\%). In our cohort, twenty-eight patients (90\%) received chemotherapy. Fifteen patients (48\%) received carboplatin/paclitaxel as their first chemotherapy, 7 patients (23\%) received ifosfamide/paclitaxel, 6 patients $(19 \%)$ received a different regimen and 3 patients (10\%) did not receive chemotherapy. Only one patient (3\%) received post-operative radiation.

Table 1 legend: Categorical variables are presented as " $n$ " (\%). Continuous variables are presented as mean \pm standard deviation. Patients in the "Other Platinum" category include those receiving Ifosfamide/Cisplatin, Carboplatin/Cytoxan, and Adriamycin/Cisplatin. P-values represent the following comparisons between the five chemotherapy groups: one-way ANOVA for mean age at diagnosis and chi-squared for stage, debulking status, 
Table 1 Demographic information by initial chemotherapy regimen with regard to age, stage, debulking surgery, and radiation

\begin{tabular}{|c|c|c|c|c|c|c|}
\hline Demographic & All & No chemo & $\begin{array}{l}\text { Carboplatin/ } \\
\text { Paclitaxel }\end{array}$ & $\begin{array}{l}\text { Ifosfamide/ } \\
\text { Paclitaxel }\end{array}$ & $\begin{array}{l}\text { Other } \\
\text { Platinum }\end{array}$ & $p$-value \\
\hline Total N & 31 & $3(10 \%)$ & $15(48 \%)$ & $7(23 \%)$ & $6(19 \%)$ & \\
\hline $\begin{array}{l}\text { Mean age at diagnosis } \\
\text { (yrs } \pm \text { St dev.) }\end{array}$ & $65.39 \pm 11.3$ & $70.67 \pm 7.6$ & $68.33 \pm 11.3$ & $59.29 \pm 4.4$ & $62.50 \pm 16.1$ & 0.257 \\
\hline \multicolumn{7}{|l|}{ STAGE } \\
\hline Stage IC-II & $7(22.6 \%)$ & $0(0 \%)$ & $4(26.7 \%)$ & $2(28.6 \%)$ & $1(16.7 \%)$ & \multirow[t]{3}{*}{0.736} \\
\hline Stage III & $21(67.7 \%)$ & $3(100 \%)$ & $9(60 \%)$ & $4(57.1 \%)$ & $5(83.3 \%)$ & \\
\hline Stage IV & $3(9.7 \%)$ & $0(0 \%)$ & $2(13.3 \%)$ & $1(12.3 \%)$ & $0(0 \%)$ & \\
\hline \multicolumn{7}{|l|}{ DEBULKING STATUS } \\
\hline Debulking Surgery & $29(93.5 \%)$ & $3(100 \%)$ & $14(93.3 \%)$ & $6(85.7 \%)$ & $6(100 \%)$ & \multirow[t]{5}{*}{0.562} \\
\hline Optimal debulking & $19(61.3 \%)$ & $1(33.3 \%)$ & 10 66.7\%) & $4(57.1 \%)$ & $4(66.6 \%)$ & \\
\hline Suboptimal debulking & $9(29.0 \%)$ & $2(66.7 \%)$ & $4(26.6 \%)$ & $2(28.6 \%)$ & $1(16.7 \%)$ & \\
\hline Unknown Status & $1(3.2 \%)$ & $0(0 \%)$ & $0(0 \%)$ & $0(0 \%)$ & $1(16.7 \%)$ & \\
\hline No Surgery Attempted & $2(6.5 \%)$ & $0(0 \%)$ & $1(6.7 \%)$ & $1(14.3 \%)$ & $0(0 \%)$ & \\
\hline Radiation & 1 & 0 & 0 & 0 & 1 & NA \\
\hline
\end{tabular}

and radiation. There were no significant differences between the chemotherapy groups for these variables.

Seventeen patients (55\%) achieved a complete response, defined as no measurable evidence of disease on imaging and clinical exam following completion of primary chemotherapy. Eighteen patients (58\%) were alive one year after diagnosis, 6 patients (19\%) two years after diagnosis and 3 patients (10\%) five years after diagnosis. The median PFS and OS by first-line chemotherapy regimen are presented in Table 2. For the entire cohort, the median progression-free survival was 9.3 months (95\% CI: 3.7-14.9 months), and the median overall survival was 19.7 months (95\% CI: 15.7-23.6 months). The one patient treated with cisplatin/Adriamycin (stage IIIC at diagnosis) had a PFS and OS of 213.2 months, and was excluded from further analysis after the application of Grubbs' test for outliers $(p<0.05)$.

Table 2 legend: PFS and OS are reported for each chemotherapy regimen as "Median (95\% confidence interval)."

Table 2 Median progression-free survival and overall survival by initial chemotherapy regimen

\begin{tabular}{llll}
\hline First line chemotherapy & $\mathrm{N}$ & Median PFS $(95 \% \mathrm{Cl})$ & Median OS (95\% Cl) \\
\hline No chemotherapy & 3 & $1.3(0.5-2.1)$ & $1.3(0.5-2.1)$ \\
Ifosfamide/Paclitaxel & 7 & $8.0(2.4-13.7)$ & $19.0(16.4-21.6)$ \\
Carboplatin/Paclitaxel & 15 & $17.8(4.0-31.6)$ & $23.2(22.0-24.4)$ \\
Ifosfamide/Cisplatin & 4 & $13.0(0.0-28.3)$ & $20.6(0.0-65.2)$ \\
Carboplatin/Cytoxan & 1 & $13.4(\mathrm{NA})$ & $13.4(\mathrm{NA})$ \\
Adriamycin/Cisplatin & 1 & $213.2(\mathrm{NA})$ & $213.2(\mathrm{NA})$ \\
Total & 31 & $9.3(3.7-14.9)$ & $19.7(15.7-23.6)$ \\
\hline
\end{tabular}

Next PFS and OS were directly compared by first-line chemotherapy regimen. Median progression-free survival for patients receiving no chemotherapy was 1.3 months (95\% CI: 0.5-2.1 months). The three patients who did not receive chemotherapy (all stage IIIC at diagnosis) were all determined to have too poor of a performance status to receive chemotherapy. Given the common clinical use of chemotherapy doublets ifosfamide/paclitaxel and carboplatin/paclitaxel based on uterine carcinosarcoma literature, we sought to compare these two specific regimens in our ovarian carcinosarcoma cohort. Kaplan-Meier curves were plotted for PFS and OS for the two treatment groups and comparison carried out with a log-rank Mantel-Cox test (Fig. 1a and b). In comparing the outcomes for carboplatin/paclitaxel as a first-line regimen versus to ifosfamide/paclitaxel, those receiving carboplatin/paclitaxel had a median PFS of 17.8 months (95\% CI: $4.0-31.6$ months) compared to a median PFS of 8.0 months (95\% CI: $2.4-13.7$ months) for ifosfamide/paclitaxel $(p=0.025)$, representing a statistically significant improvement in PFS with carboplatin/ paclitaxel as first-line chemotherapy (Fig. 1a). OS was similar between groups in this comparison with a median OS of 23.2 months (95\% CI: $22.0-24.4$ months) for carboplatin/paclitaxel and 19.0 months (95\% CI: 16.4-21.6 months) for ifosfamide/paclitaxel $(p=0.350)$ (Fig. 1b).

Kaplan-Meier curves were similarly plotted for PFS and OS for both platinum and non-platinum containing regimens (Fig. 2a and b), and they were compared with a log-rank Mantel-Cox test. In comparing platinum to non-platinum containing regimens, the median PFS for platinum-containing regimens was longer at 13.4 months compared to 8.0 months for non-platinum regimens 

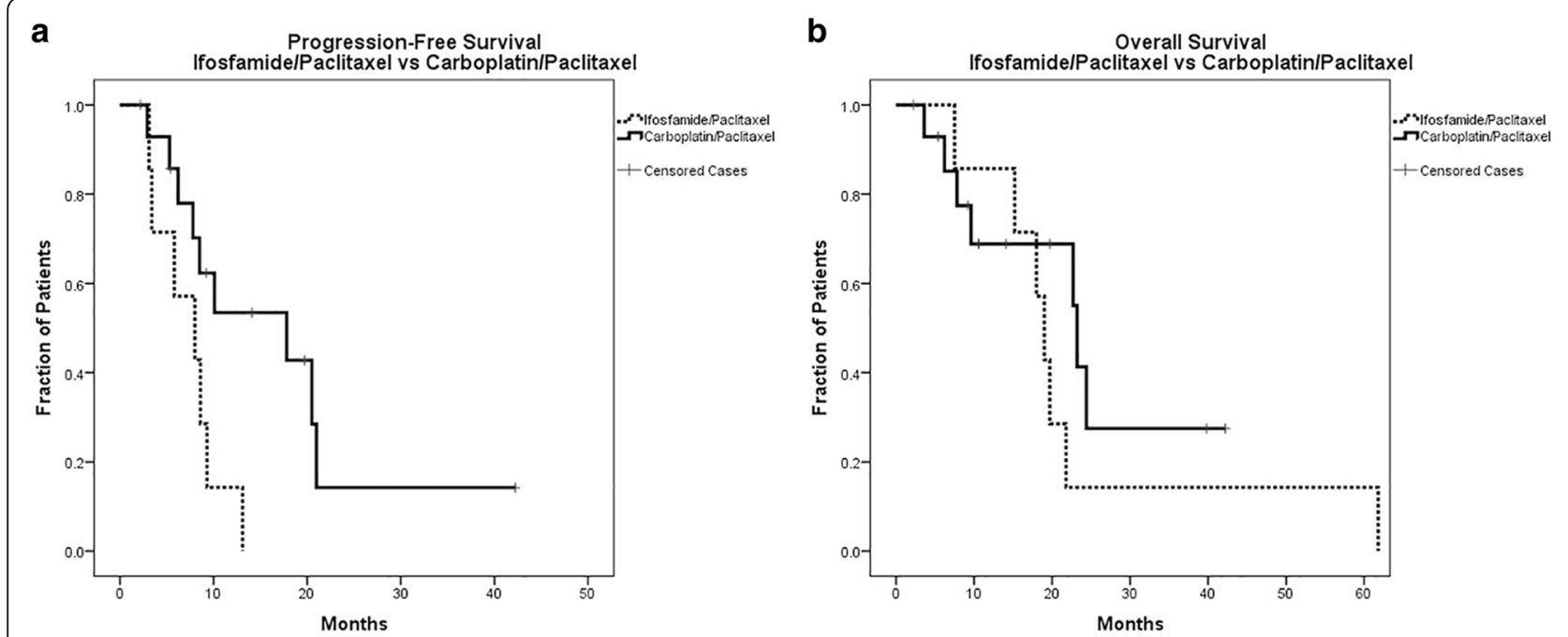

Fig. 1 Progression-Free Survival and Overall Survival for Ifosfamide/Paclitaxel vs Carboplatin/Paclitaxel. a Kaplan Meier curve comparing PFS by initial chemotherapy: Ifosfamide/Paclitaxel compared to Carboplatin/Paclitaxel. Analyzed by log-rank Mantel-Cox test. $p=0.025$. b Kaplan Meier curve comparing OS by initial chemotherapy: Ifosfamide/Paclitaxel compared to Carboplatin/Paclitaxel. Analyzed by log-rank Mantel-Cox test. $p=0.350$

$(p=0.008)$. Overall survival was similar between the two groups at a median of 22.7 months for platinumcontaining regimens and 19.0 months for non-platinumcontaining regimens $(p=0.323)$. Given the potential that the platinum component of the chemotherapy doublet is critical to the effectiveness of the regimen, we made one additional comparison: ifosfamide/cisplatin $(n=4)$ compared to carboplatin/paclitaxel $(n=15)$. There was no difference in median PFS (13.0 vs 17.8 months, $p=$ 0.750 ) or OS (20.6 vs 23.2 months, $p=0.657$ ) between these platinum-containing groups.

\section{Discussion}

This retrospective study suggests that utilizing carboplatin/paclitaxel as first-line chemotherapy improves progression-free survival in patients with ovarian carcinosarcoma when compared to ifosfamide/paclitaxel. Interestingly, comparison of the patient population in our cohort treated with carboplatin/paclitaxel compared to ifosfamide/paclitaxel revealed that the carboplatin/ paclitaxel patients were older (mean age of 68.3 years versus 59.3 years, $p=0.014$ ) yet still had a longer PFS. Otherwise, there was no statistically significant
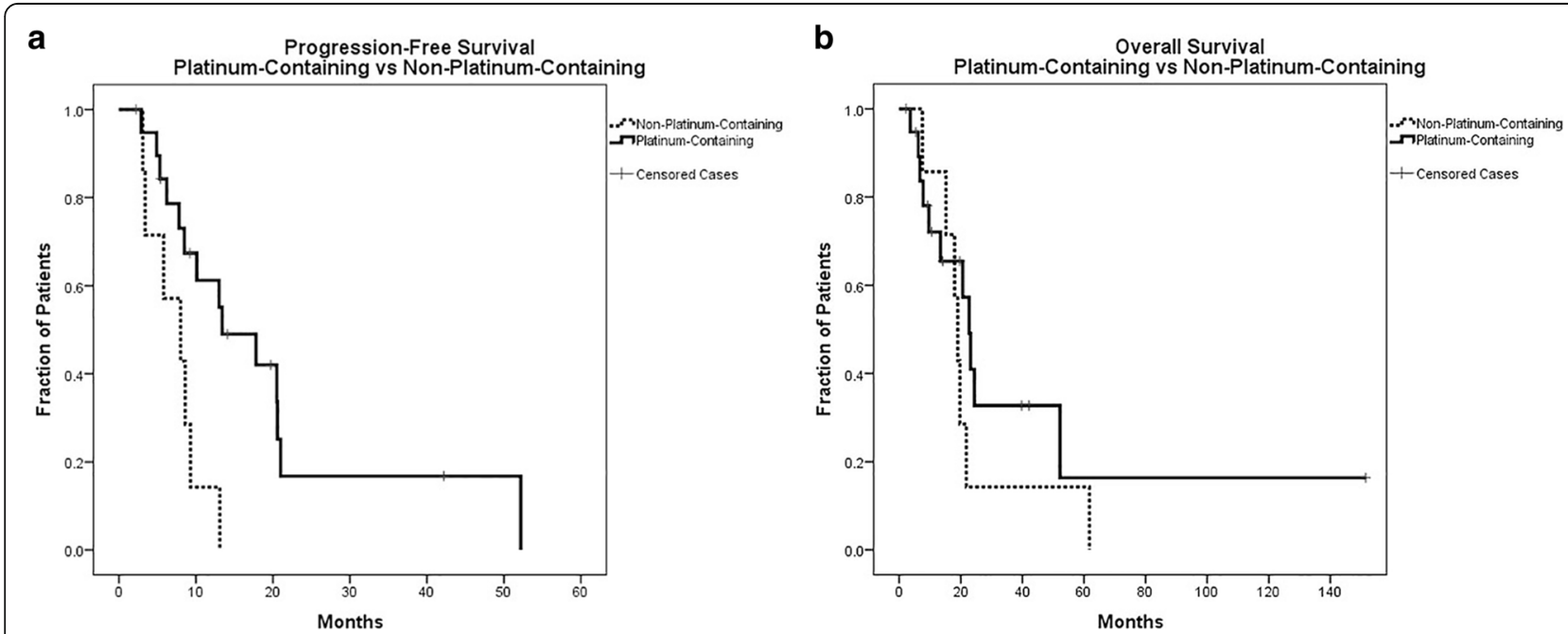

Fig. 2 Progression-Free Survival and Overall Survival for Platinum-Containing vs Non-Platinum-Containing Regimens. a Kaplan Meier curve comparing PFS by initial chemotherapy: platinum-containing compared to non-platinum-containing. Analyzed by log-rank Mantel-Cox test. $p=0.008$. b Kaplan Meier curve comparing OS by initial chemotherapy: platinum-containing compared to non-platinum-containing. Analyzed by log-rank Mantel-Cox test. $p=0.323$ 
difference between these two groups with regard to multiple variables: the percentage of patients that underwent debulking surgery (carboplatin/paclitaxel 93\% versus ifosfamide/paclitaxel $86 \%, p=0.34$ ), the percentage in whom optimal debulking was achieved (71\% versus $65 \%$, $p=0.83$ ), distribution of stage ( $60 \%$ stage III vs $57 \%, p=$ 0.736 ), or the percentage in whom complete response was achieved following initial therapy ( $80 \%$ vs $57 \%, p=$ 0.26) (Table 1).

No treatment approach queried demonstrated a superior overall survival as compared to other treatment options. We hypothesize that this is due to subsequent treatment cross-over following progression or recurrence, such that many patients receive all of the potentially active chemotherapy agents at some time during their treatment. Due to post-progression therapy impacting OS in the treatment of ovarian cancer, it has been proposed that PFS be used as a valid endpoint for the assessment of treatment efficacy [18].

The relative rarity of ovarian carcinosarcoma has limited the ability to perform prospective trials assessing chemotherapy options. A Cochrane review of chemotherapy and/or radiotherapy in combination with surgery for ovarian carcinosarcoma found no evidence to inform decisions about neoadjuvant or post-operative chemotherapy for women with ovarian carcinosarcoma [19]. Additionally, data from GOG-261, which allowed for enrollment of patients with ovarian carcinosarcoma in addition to uterine carcinosarcoma, is not yet available, and we do not know yet how many ovarian carcinosarcoma patients were enrolled in the study. Thus, we are currently limited to retrospective studies to guide treatment approaches. Multiple small studies have investigated prognosis and treatment options for ovarian carcinosarcoma (Table 3).

Table 3 legend: Studies are listed in the order they appear in the text. For each study, the number of participants, median PFS and OS, as well as key findings are listed. If the study compared different chemotherapy regimens directly, it is listed. "ND" = not determined.

Although several of these studies attempted to compare different potential chemotherapy regimens, no definitive preferred first-line treatment has emerged. In the few studies where direct comparisons between regimens were made, results were mixed. In one study, PFS was longer in patients treated with ifosfamide/cisplatin compared to carboplatin/paclitaxel [14]. However, in another, PFS was the same when comparing ifosfamide/cisplatin to carboplatin/paclitaxel [15]. Additionally, no study prior to our work directly compares ifosfamide/paclitaxel to carboplatin/paclitaxel, an especially relevant comparison given the frequent extrapolation of uterine carcinosarcoma treatment regimens to the care of women with ovarian carcinosarcoma $[9,10]$. Our study adds to the published literature by addressing this comparison. Moreover, our data further lend support to the first-line use of platinum-based regimens in ovarian carcinosarcoma, consistent with our evolving understanding

Table 3 Key retrospective studies of chemotherapy regimens in patients with ovarian carcinosarcomas

\begin{tabular}{|c|c|c|c|c|c|c|}
\hline Study & Citation & $N$ & Median PFS (mo) & Median OS (mo) & Findings & $\begin{array}{l}\text { Regimens } \\
\text { Compared }\end{array}$ \\
\hline Brown, E. et al. & {$[6]$} & 65 & 6.4 & 8.2 & $\begin{array}{l}\text { Ovarian carcinosarcomas are associated with poorer response } \\
\text { to chemotherapy, PFS and disease specific survival compared } \\
\text { to high grade papillary serous carcinoma of the ovary. } \\
\text { Debulking status significantly impacts outcomes. }\end{array}$ & No \\
\hline Kanis, M.J. et al. & [13] & 28 & 10 & 21 & $\begin{array}{l}\text { No difference in PFS and OS between patients treated with } \\
\text { carboplatin/paclitaxel and those treated with other first-line } \\
\text { chemotherapy regimens. Optimal debulking improves PFS. }\end{array}$ & Yes \\
\hline Rutledge, T.L. et al. & {$[14]$} & 31 & ND & 21 & $\begin{array}{l}\text { Advanced stage disease worsens PFS. PFS is better in } \\
\text { ifosfamide/cisplatin compared to carboplatin/paclitaxel. OS } \\
\text { similar between the two chemotherapy regimens. }\end{array}$ & Yes \\
\hline Silasi, D.A. et al. & [15] & 22 & $6-13$ & 38 & $\begin{array}{l}\text { PFS is the same for ifosfamide/cisplatin compared to } \\
\text { carboplatin/paclitaxel. }\end{array}$ & Yes \\
\hline Rauh-Hain, J.A. et al. & {$[17]$} & 50 & 11 & 24 & $\begin{array}{l}\text { Ovarian carcinosarcomas are associated with poorer response } \\
\text { to platinum-based chemotherapy, PFS and disease specific } \\
\text { survival compared to high grade papillary serous carcinoma } \\
\text { of the ovary. }\end{array}$ & No \\
\hline Cicin, I. et al. & {$[19]$} & 26 & ND & 26 & $\begin{array}{l}\text { Median survival is longer with early versus late stage disease. } \\
\text { Adjuvant platinum-based chemotherapy is predictive of better } \\
\text { outcome. }\end{array}$ & Yes \\
\hline Leiser, A.L. et al. & {$[20]$} & 30 & 12 & 43 & $\begin{array}{l}\text { Median PFS is } 12 \text { months when treated with combination of } \\
\text { a platinum and taxane. }\end{array}$ & No \\
\hline Brackmann, M. et al. & - & 31 & 9.3 & 19.7 & $\begin{array}{l}\text { Longer PFS with carboplatin/paclitaxel compared to } \\
\text { ifosfamide/paclitaxel. OS similar between comparison groups. }\end{array}$ & Yes \\
\hline
\end{tabular}


of carcinosarcomas as epithelial malignancies and previous reports supporting this approach [16, 20-25]. Given the lower morbidity of carboplatin/paclitaxel in comparison to regimens containing ifosfamide, cisplatin, or both, our comparison is of particular interest. Our work is similar in sample size to previously reported studies and PFS and OS are in line with published data.

Our study has similar limitations to previously published work, including overall low patient numbers, the retrospective nature of the work, the non-randomized treatment groups (including some shift in chemotherapy preference over time) and the high crossover rate with subsequent therapies that confounds interpretation of overall survival. Additionally, several patients in the cohort represent recent diagnoses and their survival data is not yet mature. We plan an additional analysis of these patients in the future.

As our understanding of tumor biology expands, continued molecular characterization of ovarian carcinosarcoma is warranted to define both targetable alterations and predictive biomarkers of response. Significant progress was made in this regard with the finding that ovarian and uterine carcinosarcomas represent metaplastic carcinomas, therefore suggesting traditional regimens for carcinomas would be favored over sarcoma regimens [2]. These data support the biologic rationale for treating ovarian carcinosarcoma patients with carboplatin/paclitaxel, consistent with preferred treatment for ovarian carcinomas. Detailed molecular analysis of ovarian carcinosarcomas remains lacking at this time. Studies are generally still performed with both ovarian and uterine carcinosarcomas to increase sample size, although it is unclear whether there is a molecular rationale for this approach. It is known, however, that altered expression of p53 is frequent in gynecologic carcinosarcomas [26]. A recent molecular characterization of an ovarian carcinosarcoma patient-derived xenograft revealed p53 and phosphoinositide-3-kinase, catalytic, alpha polypeptide (PIK3CA) mutations, as well as epidermal growth factor receptor overexpression, vascular endothelial growth factor receptor $\mathrm{C}$ overexpression and activation of the insulin-like growth factor pathway [27]. Further studies are warranted to determine the frequency of both germline and somatic genetic alterations in ovarian carcinosarcoma patients and the potential for such mutations to inform clinical treatment decisions.

\section{Conclusions}

In conclusion, ovarian carcinosarcomas are rare, aggressive tumors with poor prognosis. The optimal first-line chemotherapy for these patients remains unknown. In our study, patients treated with carboplatin/paclitaxel have a longer PFS than patients treated with ifosfamide/ paclitaxel. Moreover, platinum-containing regimens appear to prolong PFS when compared to non-platinum containing regimens. Overall survival was similar for all treatment groups, likely due to subsequent treatment crossover at the time of recurrence. Given lower morbidity, lower cost and fewer hospital days associated with carboplatin/paclitaxel regimens as well as longer PFS, we favor this first-line chemotherapy for ovarian carcinosarcomas after primary surgical debulking. Further study into optimal chemotherapy remains warranted, including cooperative clinical trials and continued molecular characterization of ovarian carcinosarcomas.

\section{Abbreviations}

GOG: Gynecologic Oncology Group; MMMT: Malignant mixed Mullerian tumors; ND: Not determined; OS: Overall survival; PFS: Progression-free survival; PIK3CA: Phosphoinositide-3-kinase, catalytic, alpha polypeptide; SEER: Surveillance, Epidemiology, and End Results

\section{Acknowledgments}

The authors would like to thank Emily Kobernik for her input on statistical analysis.

\section{Funding}

Funding was provided in part by the University of Michigan Department of Obstetrics and Gynecology Ansbacher Resident Research Fund to Melisa Brackmann. The funding body played no role in the design of the study, collection, analysis, and interpretation of data, or in writing the manuscript.

\section{Availability of data and materials}

The de-identified dataset generated and analyzed during the current study is not publicly available consistent with the prior the approval of the University of Michigan Institutional Review Board but are available from the corresponding author on reasonable request pending approval of the University of Michigan Institutional Review Board.

\section{Authors' contributions}

MB made substantial contributions to conception and design, acquisition of data, analysis and interpretation of data, and drafting and revising the manuscript. MS made substantial contributions to conception and design, analysis and interpretation of data, and revising the manuscript. SU made substantial contributions to conception and design, analysis and

interpretation of data, and revising the manuscript. JE made substantial contributions to acquisition of data and revising the manuscript. RKR made substantial contributions to conception and design, analysis and interpretation of data, and revising the manuscript. KM made substantial contributions to conception and design, acquisition of data, analysis and interpretation of data, and drafting and revising the manuscript. All authors read and approved the final manuscript.

\section{Ethics approval and consent to participate}

This study was approved by the University of Michigan Institutional Review Board (IRB \# HUM00099459). This study was determined not to require review by the University of Michigan Health System Comprehensive Cancer Center Protocol Review Committee as it did not involve a cancer-related therapeutic clinical research protocol or a cancer prevention or control research protocol. A waiver of informed consent was granted based on the following University of Michigan Institutional Review Board criteria all being met: 1 . The research involved no more than minimal risk to the subjects; 2. The waiver would not adversely affect the rights and welfare of the subjects; 3 . The research could not practicably (i.e., feasibly) be carried out without the waiver or alteration (given the retrospective nature spanning over 20 years during which many patients died); 4. Whenever appropriate, it was agreed that the subjects would be provided with additional pertinent information obtained from the study. 


\section{Competing interests}

The authors declare that they have no competing interests.

\section{Publisher's Note}

Springer Nature remains neutral with regard to jurisdictional claims in published maps and institutional affiliations.

\section{Received: 6 September 2017 Accepted: 31 January 2018}

\section{Published online: 09 February 2018}

\section{References}

1. Pacaut C, Bourmaud A, Rivoirard R, Moriceau G, Guy JB, Collard O, Bosacki C, Jacquin JP, Levy A, Chauleur C, et al. Uterine and ovary carcinosarcomas: outcome, prognosis factors, and adjuvant therapy. Am J Clin Oncol. 2015; 38(3):272-7.

2. Schipf A, Mayr D, Kirchner T, Diebold J. Molecular genetic aberrations of ovarian and uterine carcinosarcomas-a CGH and FISH study. Virchows Arch. 2008;452(3):259-68.

3. Yamada SD, Burger RA, Brewster WR, Anton D, Kohler MF, Monk BJ. Pathologic variables and adjuvant therapy as predictors of recurrence and survival for patients with surgically evaluated carcinosarcoma of the uterus. Cancer. 2000;88(12):2782-6.

4. Rauh-Hain JA, Diver EJ, Clemmer JT, Bradford LS, Clark RM, Growdon WB, Goodman AK, Boruta DM 2nd, Schorge JO, del Carmen MG. Carcinosarcoma of the ovary compared to papillary serous ovarian carcinoma: a SEER analysis. Gynecol Oncol. 2013;131(1):46-51.

5. Rauh-Hain JA, Birrer M, Del Carmen MG. Carcinosarcoma of the ovary, fallopian tube, and peritoneum: prognostic factors and treatment modalities. Gynecol Oncol. 2016;142(2):248-54.

6. Brown E, Stewart M, Rye T, Al-Nafussi A, Williams AR, Bradburn M, Smyth J, Gabra H. Carcinosarcoma of the ovary: 19 years of prospective data from a single center. Cancer. 2004;100(10):2148-53.

7. George EM, Herzog TJ, Neugut Al, Lu YS, Burke WM, Lewin SN, Hershman DL, Wright JD. Carcinosarcoma of the ovary: natural history, patterns of treatment, and outcome. Gynecol Oncol. 2013;131(1):42-5.

8. Mano MS, Rosa DD, Azambuja E, Ismael G, Braga S, D'Hondt V, Piccart M, Awada A. Current management of ovarian carcinosarcoma. Int J Gynecol Cancer. 2007;17(2):316-24.

9. Homesley HD, Filiaci V, Markman M, Bitterman P, Eaton L, Kilgore LC, Monk BJ, Ueland FR, Gynecologic Oncology G. Phase III trial of ifosfamide with or without paclitaxel in advanced uterine carcinosarcoma: a gynecologic oncology group study. J Clin Oncol. 2007;25(5):526-31.

10. Galaal K, van der Heijden E, Godfrey K, Naik R, Kucukmetin A, Bryant A, Das $\mathrm{N}$, Lopes AD. Adjuvant radiotherapy and/or chemotherapy after surgery for uterine carcinosarcoma. The Cochrane database of systematic reviews. 2013; 2:CD006812.

11. Powell MA, Filiaci VL, Rose PG, Mannel RS, Hanjani P, Degeest K, Miller BE, Susumu N, Ueland FR. Phase II evaluation of paclitaxel and carboplatin in the treatment of carcinosarcoma of the uterus: a gynecologic oncology group study. J Clin Oncol. 2010;28(16):2727-31.

12. Lacour RA, Euscher E, Atkinson EN, Sun CC, Ramirez PT, Coleman RL, Brown J, Gano JB, Burke TW, Ramondetta LM. A phase II trial of paclitaxel and carboplatin in women with advanced or recurrent uterine carcinosarcoma. International journal of gynecological cancer : official journal of the International Gynecological Cancer Society. 2011;21(3):517-22.

13. Kanis MJ, Kolev V, Getrajdman J, Zakashansky K, Cohen C, Rahaman J. Carcinosarcoma of the ovary: a single institution experience and review of the literature. Eur J Gynaecol Oncol. 2016;37(1):75-9.

14. Rutledge TL, Gold MA, McMeekin DS, Huh WK, Powell MA, Lewin SN, Mutch DG, Johnson GA, Walker JL, Mannel RS. Carcinosarcoma of the ovary-a case series. Gynecol Oncol. 2006;100(1):128-32.

15. Silasi DA, Illuzzi JL, Kelly MG, Rutherford TJ, Mor G, Azodi M, Schwartz PE. Carcinosarcoma of the ovary. International journal of gynecological cancer : official journal of the International Gynecological Cancer Society. 2008;18(1): 22-9.

16. Duska LR, Garrett A, Eltabbakh GH, Oliva E, Penson R, Fuller AF. Paclitaxel and platinum chemotherapy for malignant mixed mullerian tumors of the ovary. Gynecol Oncol. 2002;85(3):459-63.

17. Rauh-Hain JA, Growdon WB, Rodriguez N, Goodman AK, Boruta DM 2nd, Schorge JO, Horowitz NS, del Carmen MG. Carcinosarcoma of the ovary: a case-control study. Gynecol Oncol. 2011;121(3):477-81.
18. Herzog TJ, Armstrong DK, Brady MF, Coleman RL, Einstein MH, Monk BJ, Mannel RS, Thigpen JT, Umpierre SA, Villella JA, et al. Ovarian cancer clinical trial endpoints: Society of Gynecologic Oncology white paper. Gynecol Oncol. 2014;132(1):8-17.

19. Shylasree TS, Bryant A, Athavale R. Chemotherapy and/or radiotherapy in combination with surgery for ovarian carcinosarcoma. The Cochrane database of systematic reviews. 2013;2:CD006246.

20. Cicin I, Saip P, Eralp Y, Selam M, Topuz S, Ozluk Y, Aydin Y, Topuz E. Ovarian carcinosarcomas: clinicopathological prognostic factors and evaluation of chemotherapy regimens containing platinum. Gynecol Oncol. 2008;108(1): 136-40.

21. Leiser AL, Chi DS, Ishill NM, Tew WP. Carcinosarcoma of the ovary treated with platinum and taxane: the memorial Sloan-Kettering cancer center experience. Gynecol Oncol. 2007;105(3):657-61.

22. Loizzi V, Cormio G, Camporeale A, Falagario M, De Mitri P, Scardigno D, Putignano G, Selvaggi LE. Carcinosarcoma of the ovary: analysis of 13 cases and review of the literature. Oncology. 2011;80(1-2):102-6.

23. Inthasorn P, Beale P, Dalrymple C, Carter J. Malignant mixed mullerian tumour of the ovary: prognostic factor and response of adjuvant platinumbased chemotherapy. Aust N Z J Obstet Gynaecol. 2003;43(1):61-4.

24. Sit AS, Price FV, Kelley JL, Comerci JT, Kunschner AJ, Kanbour-Shakir A, Edwards RP. Chemotherapy for malignant mixed Mullerian tumors of the ovary. Gynecol Oncol. 2000;79(2):196-200.

25. Jernigan AM, Fader AN, Nutter B, Rose P, Tseng JH, Escobar PF. Ovarian carcinosarcoma: effects of cytoreductive status and platinum-based chemotherapy on survival. Obstet Gynecol Int. 2013;2013:490508.

26. Costa MJ, Vogelsan J, Young $\sqcup$ J. p53 gene mutation in female genital tract carcinosarcomas (malignant mixed mullerian tumors): a clinicopathologic study of 74 cases. Mod Pathol. 1994;7(6):619-27.

27. Glaser G, Weroha SJ, Becker MA, Hou X, Enderica-Gonzalez S, Harrington SC, Haluska P. Conventional chemotherapy and oncogenic pathway targeting in ovarian carcinosarcoma using a patient-derived tumorgraft. PLOS One. 2015:10(5):e0126867.

\section{Submit your next manuscript to BioMed Central and we will help you at every step:}

- We accept pre-submission inquiries

- Our selector tool helps you to find the most relevant journal

- We provide round the clock customer support

- Convenient online submission

- Thorough peer review

- Inclusion in PubMed and all major indexing services

- Maximum visibility for your research

Submit your manuscript at www.biomedcentral.com/submit
Biomed Central 